\title{
Uterine Rupture in Subsequent Pregnancy in a Patient with Previous Uterine Manipulator Associated Uterine Perforation
}

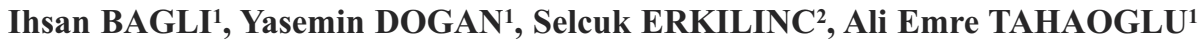 \\ Diyarbakir, Turkey
}

\begin{abstract}
To report a case who experienced uterine rupture at 38th week of gestation and had a history of manipulator associated uterine rupture.

A 25 years old primigravid woman at 38 weeks of gestation was referred to our hospital with the signs of active labor. Heavy lower abdominal cramps and signs of acute abdomen suggested uterine rupture and emergent cesarean section was performed. A $3 \mathrm{~cm}$ in size and circular in shape uterine rupture at the fundus that was imitative of enlarged previous manipulator associated rupture was observed.
\end{abstract}

A manipulator associated uterine rupture should be repaired when encountered in reproductive ages in order to prevent a possible uterine perforation during subsequent pregnancy.

Keywords: Uterine rupture, Pregnancy, Damaged by uterine manipulators

Gynecol Obstet Reprod Med 2019;25(3):174-175

\section{Introduction}

Uterine rupture during pregnancy is a catastrophic obstetric complication that may cause maternal and fetal mortality and serious morbidity. Risk factors associated with uterine rupture were reported to be previous cesarean section, myomectomy, dilatation curettage and operative hysteroscopy (1). Spontaneous uterine rupture is a rare event during pregnancy. Tip of manipulator may cause uterine rupture when proper insertion is not carried out. Since perforation during

${ }^{1}$ University of Health Sciences Diyarbakir Gazi Yasargil Research and Training Hospital, Department of Obstetrics and Gynecology, Diyarbakir, Turkey

${ }^{2}$ University of Health Sciences, Tepecik Research and Training Hospital, Department of Gynecologic Oncology Izmir, Turkey

Address of Correspondence: Ihsan Bagli

University of Health Sciences

Diyarbakir Gazi Yasargil Research and

Training Hospital, Department of

Obstetrics and Gynecology,

21070 Diyarbakir, Turkey

ihsanbagli@gmail.com

Submitted for :Publication:

30.06 .2018

Revised for: Publication:

31.07.2018

Accepted for Publication:

03.10 .2018

ORCID IDs of the authors:

IB: 0000-0002-3195-9164,

SE:0000-0002-6512-9070, $Y D: 0000-0002-2614-4411$,

AET:0000-0002-3460-3468

\begin{tabular}{|c|c|}
\hline \multirow{3}{*}{ 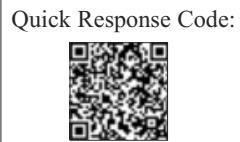 } & Access this article online \\
\hline & $\begin{array}{l}\text { Website: www.gorm.com.tr } \\
\text { e- mail: info@gorm.com.tr }\end{array}$ \\
\hline & DOI:10.21613/GORM.2018.813 \\
\hline
\end{tabular}

How to cite this article: Bagli I. Dogan Y. Erkilinc S. Tahaoglu AE. Uterine Rupture in Subsequent Pregnancy in a Patient with Previous Uterine Manipulator Associated Uterine Perforation. Gynecol Obstet Reprod Med. 2019;25(3):174-175 uterine manipulations mostly associated with minimal amount of hemorrhage, expectant management is acceptable.

In this report, we aimed to present a case that had a history of previous uterine perforation during uterine manipulation and had uterine rupture during labor. To the best of our knowledge our manuscript is the first to report a case that experienced uterine rupture in pregnancy after manipulator associated uterine perforation.

\section{Case Report}

A 25 years old primigravid woman at 38 weeks of gestation was referred to our hospital with signs of active labor. The patient underwent laparoscopic chromopertubation for the investigation of primary infertility. Uterine rupture occurred during insertion of the tip of RUMI II manipulator system. Insignificant bleeding at the site of rupture occurred and suturing was not performed. The patient was hemodynamically stable and discharged at postoperative second day without any sign of intra-abdominal bleeding. A spontaneous pregnancy occurred 2 months after the operation. No problem occurred during pregnancy follow up. The patient was admitted to our department with heavy lower abdominal cramps and the signs of acute abdomen suggesting uterine rupture. Amniotic membrane was intact cervical effacement and dilatation were $70 \%$ and $2 \mathrm{~cm}$ respectively. The total amniotic fluid index was 80 $\mathrm{mm}$ in four quadrants and biometric measurements were concordant with the age of gestation. Blood pressure was 110/70 $\mathrm{mmHg}$, heart rate was $98 / \mathrm{min}$. Signs of acute fetal distress and recent onset of anhydramnios were observed during the ultrasound examination. Emergent cesarean section was performed with the suspicion of uterine rupture and $1000 \mathrm{~mL}$ of blood 
was observed in the peritoneal cavity. A circular uterine perforation $3 \mathrm{~cm}$ in size at the uterine fundus that an enlarged imitative of previous uterine rupture caused by uterine manipulator was detected (Figure 1). Newborn was healthy and had Apgar scores of 8 and 9 at $1^{\text {st }}$ and $5^{\text {th }}$ minutes, respectively. The site of perforation was repaired with continued 1-0 polyglicolic acid sutures. Pre-operative and postoperative hemoglobin levels were $13.6 \mathrm{~g} / \mathrm{dL}$ and $9.4 \mathrm{~g} / \mathrm{dL}$ respectively. The patient was discharged at $2^{\text {nd }}$ day after cesarean section. The patient was examined on $7^{\text {th }}$ day after the operation and showed normal clinical, ultrasound and laboratory findings.

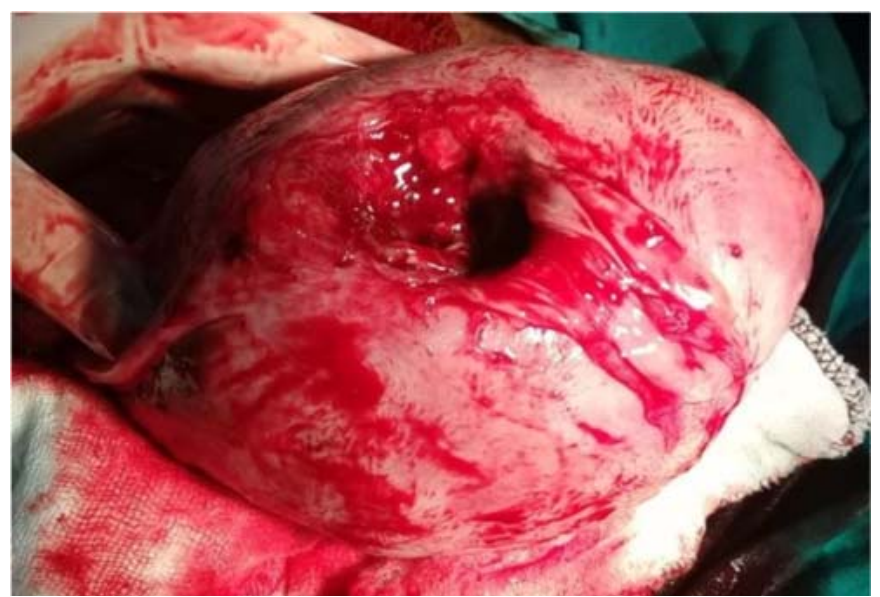

Figure 1: A circular shaped uterine perforation that an enlarged model of previous uterine rupture can be seen at uterine fundus

\section{Discussion}

Uterine rupture in pregnancy may occur spontaneously or by trauma. The overall incidence of rupture of the pregnant uterus was reported to be $1: 2428$ deliveries $(0.04 \%)(2)$. The presence of a myometrial surgical incision on the uterine wall is known to be the major risk factor for uterine rupture. Other risk factors include grand multiparity, extreme use of uterotonic drugs, prolonged labor, uterine abnormalities, dystocia, abnormal placentation, history of dilatation and curettage and hysteroscopy $(3,4)$. Nevertheless, uterine rupture caused by uterine manipulator was reported to be rare (5). Risk factors for uterine rupture in subsequent pregnancy after gynecologic surgery were investigated by Chao et al. However, manipulator associated uterine rupture was not found to be a risk factor for subsequent uterine rupture during pregnancy (6). Uterine rupture should be taken into consideration when signs of acute abdomen occur even in a primigravid patient. Although chromopertubation with the RUMI manipulator is a simple procedure during laparoscopic surgery, uncontrolled insertion of the tip of manipulator may result in perforation on the uterine wall. Silent or highly suspected uterine perforation during curettage or hysteroscopy was reported to be associated with uterine rupture in subsequent pregnancies (2). Expectant follow up of patient is the most preferred management when uterine rupture encountered during uterine manipulation. Although expectant management is regarded to be safe, the condition may result in significant obstetrical morbidity. However, the absolute risk of uterine rupture during active labor after manipulator associated uterine perforation is not known. The possible explanation of the rupture in a gravid uterus after manipulator associated uterine perforation may be the short time interval between previous operation and active labor. Insufficient tissue regeneration and less tissue strength that were caused by earlier conception after uterine manipulator associated rupture were the possible causes of uterine perforation in the current case report. Although evidence suggests expectant management of uterine perforation caused by the uterine manipulator, our case report showed the importance of repairing the rupture in order to prevent a subsequent uterine perforation during pregnancy. Reporting manipulator associated uterine rupture in operative notes is of importance in order to be aware of a possible uterine perforation during pregnancy. Patients should be warned against earlier conception that may be associated with subsequent uterine rupture during pregnancy.

A manipulator associated uterine rupture should be repaired when encountered in reproductive ages in order to prevent a possible uterine perforation during subsequent pregnancy.

Author Contrubitions: IB: Concept, data processing, analysis, and interpretation, writer. YD: Data collection, processing, analysis, and interpretation. AET: Supervision, concept, design, data collection and processing, statistical analysis and data interpretation. SE: Supervision, concept, design, analysis and interpretation.

\section{References}

1. Uccella S, Cromi A, Bogani G, Zaffaroni E, Ghezzi F Spontaneous prelabor uterine rupture in a primigravida: A case report and review of the literature. Am J Obstet Gynecol. 2011;205(5):e6-8.

2. Sayed Ahmed WA, Habash YH, Hamdy MA, Ghoneim HM. Rupture of the pregnant uterus -a 20-year review. J Matern Fetal Neonatal Med. 2017;30(12):1488-93.

3. Reed WC. Large uterine defect found at cesarean section: a case report. J Reprod Med. 2003;48(1):60-2.

4. Koutélé F, Nicolas K, Rivière MF, Ledoux A. About two cases of uterine rupture outside of labour: consequences of an unnoticed previous perforation. Gynecol Obstet Fertil. 2003;31(4):362-4.

5. Van den Haak L, Alleblas C, Nieboer TE, Rhemrev JP, Jansen FW. Efficacy and safety of uterine manipulators in laparoscopic surgery: a review. Arch Gynecol Obstet. 2015; 292(5):1003-11.

6. Chao AS, Chang YL, Yang LY, Chao A, Chang WY, Su SY et al. Laparoscopic uterine surgery as a risk factor for uterine rupture during pregnancy. PLoS One. 2018;13 (5):e0197307. 